\title{
TWO CONCEPTIONS OF SIMILARITY
}

\author{
BEN BLUMSON
}

\begin{abstract}
There are at least two traditional conceptions of numerical degree of similarity. According to the first, the degree of dissimilarity between two particulars is their distance apart in a metric space. According to the second, the degree of similarity between two particulars is a function of the number of (sparse) properties they have in common and not in common. This paper argues that these two conceptions are logically independent, but philosophically inconsonant.
\end{abstract}

Keywords: similarity, resemblance, properties, distance, metric spaces.

1.

There are at least two traditional conceptions of numerical degree of similarity. According to the first conception, the degree of similarity between particulars is a function of their number of (sparse) properties in common and not in common. This conception has its home in debates over the metaphysics of properties (see especially Armstrong, 1978b, 97-98, Oliver, 1996, 52 and Rodriguez-Pereyra, 2002, 65-69), but is also found in the debate over the resemblance theory of pictorial representation (Blumson, 2014, 179-198).

According to the second, the degree of dissimilarity between particulars is their distance apart in a metric space. This conception has its home in

Date: January 11, 2018. 
psychology and the social sciences (see especially Suppes et al., 1989, 159207), but also has applications in philosophy - in, for example, the semantics of counterfactuals (Sobel, 1970, 430-44; Lewis, 1973, 50-2), the interpretation of probability (Bigelow, 1976; 1977), and the measurement of likeness to truth (Niiniluoto, 1987, 1-17; Oddie, 1986, 34-8).

It would be natural to assume that the two conceptions are equivalent. But I will argue that they are logically independent - they are neither equivalent nor inconsistent, and neither one entails the other. Moreover, I will argue that they are philosophically inconsonant - some intuitively plausible analyses in terms of number of properties do not define distance functions, and some intuitively plausible distance functions don't correspond to any analysis in terms of number of properties.

At least two natural analyses of dissimilarity as a function of number of properties in common and number of properties not in common do entail that dissimilarity is distance in a metric space. According to the first, the degree of dissimilarity between two particulars is their total number of properties not in common or, in other words, the sum of the number of properties the first has not in common with the second and the number of properties the second has not in common with the first.

According to the second, the degree of dissimilarity between two particulars is one minus their degree of similarity, where their degree of similarity is their proportion of properties in common or, in other words, the number of properties they have in common, divided by the number of properties they 
have in total. Even though the second analysis is more plausible than the first, I will argue that it's still inconsonant with the conception of degree of dissimilarity as distance.

To see the importance of the issue, consider an analogy with probability. One conception of probability is embodied in the axioms of the probability calculus, and it is an advantage of some analyses of probability - such as the analysis of probabilities as finite frequencies - that they entail the axioms. Likewise, one conception of dissimilarity is embodied in the definition of a metric space, and it is an advantage of some analyses of dissimilarity - such as the analyses as proportion or number of properties not in common - that they entail degree of dissimilarity is distance in a metric space.

But many of the analyses discussed in the paper do not entail that degree of dissimilarity is distance in a metric space. Moreover, just as the analysis of probability as finite frequency is inconsistent with other aspects of our ordinary conception of probability (Hajek, 1996), I will argue in section (10) that the analysis of degree of similarity as proportion of properties in common, which does entail that dissimilarity is distance in a metric space, is inconsistent with other aspects of our ordinary conception of similarity.

The relationship between properties and resemblance is a central topic in metaphysics. David Armstrong, for example, describes it as one of '...the compulsory questions of the philosophical exam paper' Armstrong, 1997, 102). By raising this traditional topic in the context of metric spaces and degrees of dissimilarity, I hope to lend greater clarity and precision to the 
topic, just as the probability calculus can be employed to lend clarity and precision to traditional debates on chance and credence.

2 .

According to the first conception, the degree of similarity between two particulars is a function of their number of (sparse) properties in common and not in common. Peas in a pod, for example, resemble each other to a high degree because they have many properties in common and few properties not in common, whereas a raven and a writing desk resemble each other to a low degree because they have few properties in common and many properties not in common.

The plausibility of this conception of degree of similarity depends on the conception of properties with which it is combined. According to abundant conceptions of properties, there is a property corresponding to every class of (possible) individuals. Abundant conceptions of properties are inappropriate to feature in analyses of degree of similarity in terms of number of properties in common and not in common - although a raven and a writing desk, for example, belong to the class of ravens and writing desks, this is not sufficient for any degree of similarity between them (Lewis, 1983,$346 ; 1986,59-60)$.

The sparse properties, in contrast, are supposed to be those which make for similarity. As David Lewis, for example, writes 'Sometimes we conceive of properties as abundant, sometimes as sparse. The abundant properties may be ... as gruesomely gerrymandered, as miscellaneously disjunctive, as you 
please. ... Sharing of them has nothing to do with similarity. ... The sparse properties are another story. Sharing of them makes for qualitative similarity...' (Lewis, 1986, 59-60). So it's sparse properties which are appropriate to feature in the analysis of degree of similarity.

In addition to making for similarity, sparse properties are also supposed to have several other roles. For example, as well as being the properties that make for similarity, they are also supposed to be the properties relevant to causation Armstrong, 1978b; Lewis, 1983, 347). But I am very sympathetic to the possibility that no one conception can play all the roles which sparse properties have been asked to (Schaffer, 2004; Dorr and Hawthorne, 2013). So in this paper, I focus exclusively on the role of sparse properties in explicating similarity.

Three clarifications. First, some authors maintain that as well as resemblances between particulars, there are also resemblance between the (sparse) properties themselves - red, for example, is supposed to resemble orange more than blue (Armstrong, 1978b, 101-15). The topic of similarity between properties raises many questions which are closely related to the questions raised in this paper (see, for example, Eddon, 2006). Nevertheless, this paper is exclusively concerned with similarity between particulars.

Second, instead of the binary distinction between sparse and abundant properties, some authors prefer a distinction which admits of degree, between the more or less natural or important properties (Lewis, 1983, 347; 1986 , 61). In this case, it's natural to analyse degree of similarity as a function of 
the weights of their properties not in common, with greater weight given to properties which are more natural or important (see, for example, Goodman, 1972, 445; Suppes et al., 1989, 207-225; Oddie, 1986, 45; Kroedel and Huber, 2013, 459-462). I discuss this analysis at length elsewhere.

Third, if the number of possible individuals is infinite, then for any two individuals, both are in infinitely many classes of possible individuals the other is in, and each is in infinitely many classes of possible individuals the other is not in Goodman, 1972, 445; Lewis, 1983, 346; 1986, 59-60). So if the number of possible particulars is infinite and properties are abundant, then everything has infinitely many properties in common and not in common with everything else, regardless of their degree of dissimilarity.

But if properties are sparse, then it doesn't follow that the number of properties in common and not in common between any pair of individuals is infinite, since it may be that genuine sparse properties correspond to only finitely many of the classes they are in Armstrong, 1989, 40). Even if the number of sparse properties is infinite, it doesn't follow that the number any particular has is infinite - even if there are infinitely many mass properties, for example, each particular still has only one mass.

In any case, I want to put this problem aside in this paper and focus on cases in which the number of properties in common and not in common between each pair of individuals is finite. As we will see in the next section, the conception of degree of similarity as distance between pairs of particulars in a metric space has its own problems with infinity. I want to know whether 
the two conceptions of similarity can cohere together at least in the finite case, before I consider the additional problems which arise in the infinite case.

3.

According to the second conception, the degree of similarity between two (possible) particulars is their distance in a metric space. A metric space is an ordered pair $\langle A, \delta\rangle$ of a set $A$ and a distance function $\delta$ (Suppes et al. 1989, 46), where a distance function $\delta: A \times A \rightarrow \mathbb{R}$ is a function such that for all $a, b, c \in A$ :

(1) $\delta(a, a)=0$

(2) $\delta(a, b)>0$ if $a \neq b$

(3) $\delta(a, b)=\delta(b, a)$ (symmetry)

(4) $\delta(a, b)+\delta(b, c) \geq \delta(a, c)$ (the triangle inequality).

In space, for example, (1) every point is at distance zero from itself, (2) all distinct points are at a positive distance from each other, (3) the distance from point $a$ to point $b$ is the same as the return distance from point $b$ to $a$, and (4) the distance from point $a$ to point $c$ via point $b$ is always at least as great as the distance from point $a$ directly to point $c$.

The paradigm example of a metric space is the distance between points in space, but there are many other examples. In particular, if we imagine particulars laid out in space in such a way that the more dissimilar they are to each other the further apart they are from each other, then we can think 
of their degree of dissimilarity as being measured by their distance apart in that space. The vividness of this analogy between dissimilarity and spatial distance is an important philosophical motivation for conceiving of degree of dissimilarity as being distance in a metric space.

Two clarifications. First, according to condition (2), there is always a positive distance between distinct particulars. But it's controversial whether there is always a difference between distinct particulars. Moreover, if properties are very sparse, then it is especially plausible that some distinct particulars have all the same properties in common and not in common, and so especially plausible that some distinct particulars have no difference between them (Armstrong, 1978a, 92-93).

In this case condition (2) must be weakened to $\delta(a, b) \geq 0$ if $a \neq b$, and the resulting space $\langle A, \delta\rangle$ is known as a pseudometric. Nevertheless, being zero distance apart or exactly alike is then an equivalence relation, which divides $A$ into equivalence classes of exactly alike particulars, and the function from each pair of equivalence classes to the distance between their elements defines a genuine metric space (Suppes et al., 1989, 47). So for the rest of the paper, I will treat exactly alike particulars as if they are identical.

Second, if the degree of dissimilarity between particulars is representable by a distance function in a metric space, then there cannot be more degrees of dissimilarity than there are real numbers. But if there are more (possible) particulars than there are real numbers, and if they are all dissimilar to 
different degrees, then there may be more degrees of dissimilarity than there are real numbers (Lewis, 1973, 51; Williamson, 1988, 458-459).

Nevertheless, I want to put this problem aside in this paper and focus on cases in which the number of different (possible) particulars is finite. As we saw in the last section, the conception of degree of similarity as a function of number of properties in common and number of properties not in common has its own problems with infinity. I want to know whether the two conceptions of similarity can cohere together at least in the finite case, before I consider the additional problems which arise in the infinite case.

4 .

According to the simplest analysis of degree of similarity as a function of number of properties in common and not in common, the degree of similarity between two particulars is simply the number of properties they have in common (see especially Gonzalez Rodriguez-Pereyra $(2002,65-69)$ for defence of this analysis). If the properties of peas in a pod were just greenness, roundness and yuckiness, for example, then their degree of similarity would be three.

In this case, it's natural to analyse degree of dissimilarity as a strictly decreasing function of degree of similarity - in other words, it's natural to assume that the higher the degree of similarity between two things, the lower their degree of dissimilarity. (Note Rodriguez-Pereyra is not committed to this analysis - he may prefer one of the analyses below. For criticism of the 
assumption that degree of dissimilarity is a strictly decreasing function of degree of similarity, see Tversky, 1977, 339-40.)

For illustrative purposes, suppose, for example, that the degree of dissimilarity between two particulars is the highest number of properties of any particular, minus the number of properties the two particulars have in common. If the highest number of properties any particular has is three, for example, then the degree of dissimilarity of peas in a pod would be three minus three, or zero.

This analysis meets condition (2), according to which the distance between two different particulars is greater than zero, since two different particulars cannot have as many as the highest number of properties of any particular in common - otherwise one of the two particulars, in order to have more properties with which to differ with the other on, would have to have more properties than the highest number of properties that any particular has, which is impossible.

Likewise, it meets condition (3), symmetry, since the number of properties $a$ has in common with $b$ is the same as the number of properties $b$ has in common with $a$, so the highest number of properties that any particular has minus the number of properties $a$ has in common with $b$ is the same as the highest number of properties that any particular has minus the number of properties $b$ has in common with $a$.

Moreover, condition (3) symmetry is satisfied by any decreasing function of number of properties in common, since because the number of properties 
$a$ has in common with $b$ is the same as the number of properties $b$ has in common with $a$, and every function has only one output for each input, any function of number of properties in common between two particulars will have the same output for $b$ and $a$ as it does for $a$ and $b$.

Finally, the sum of the number of properties $a$ has in common with $b$ and the number of properties $b$ has in common with $c$ can exceed the highest number of properties that any particular has only by the number of properties $b$ has in common with both $a$ and $c$. So the number of properties $a$ has in common with $c$ is at least as great as the sum of the number of properties $a$ has in common with $b$ and the number of properties $a$ has in common with $c$, minus the highest number of properties that any particular has.

It follows that the highest number of properties any particular has minus the number $a$ has in common with $b$ plus the highest number any particular has minus the number $b$ has in common with $c$ is at least as great as the highest number any particular has minus the number $a$ has in common with $c$. In other words, the analysis of degree of dissimilarity as the highest number of properties any particular has minus number of properties in common satisfies condition (4), the triangle inequality.

However, unless all particulars have the same number of properties, the analysis does not satisfy condition (1), according to which the distance between every particular and itself is zero. If, for example, the highest number of properties of any particular were three, because the properties of a pea are greenness, roundness and yuckiness, but the properties of a chickpea were 
just brownness and roundness, because chickpeas have no taste, then the degree of dissimilarity of the chickpea to itself would be three minus two, which is greater than zero (compare Paseau, 2012, 378).

Moreover, unless all particulars have the same number of properties, condition (1) is not satisfied by any strictly decreasing function of number of properties in common, since if two particulars have different numbers of properties, then they will have different numbers of properties in common with themselves, and so a strictly decreasing function of number of properties in common would assign the particular with more properties a lesser degree of dissimilarity to itself, and so could not assign both particulars a zero degree of dissimilarity to themselves.

So analysing degree of dissimilarity as a decreasing function of number of properties in common does not entail that degree of dissimilarity is distance in a metric space. Moreover, if different particulars have different numbers of properties, then analysing degree of dissimilarity as a strictly decreasing function of degree of similarity entails that degree of dissimilarity is not distance in a metric space, and the two conceptions of degree of similarity are inconsonant with each other.

\section{5.}

A more direct way of analysing degree of dissimilarity as a function of properties in common or not in common is to define the degree of dissimilarity between two particulars as the number of properties the first has not in 
common with the second. If a pea has two properties - greenness and yuckiness - a chickpea doesn't have, for example, then the degree of dissimilarity of the pea to the chickpea according to this analysis would be two.

This analysis of degree of dissimilarity does satisfy condition (1) in the definition of a metric space, since no particular has any properties not in common with itself, and so the analysis entails that the degree of dissimilarity between every particular and itself is zero. Since a chickpea, for example, has none of its properties not in common with itself, the degree of dissimilarlity between a chickpea and itself is guaranteed to be zero.

And it satisfies condition (4), the triangle inequality. For for any property $a$ has not in common with $c$, either $a$ also has that property not in common with $b$, or else $a$ does have that property in common with $b$, but then $b$ has that property not in common with $c$, and so the sum of the number of properties $a$ has not in common with $b$ and the number of properties $b$ has not in common with $c$ must be greater than or equal to the number of properties $a$ has not in common with $c$.

But degree of dissimilarity analysed in this way still does not correspond to distance in a metric space, since it may not satisfy condition (3), symmetry. Although the degree of dissimilarity of the pea in the pod to the chickpea, for example, is two, since the pea has two properties - greenness and yuckiness - that the chickpea doesn't have, the degree of dissimilarity of the chickpea to the pea is only one, since because the chickpea has no taste, it only has one property - brownness - that the pea doesn't have. 
Moreover, since the number of properties a particular has not in common with another particular can never be less than zero, this analysis meets the weakened version of condition (2), according to which $\delta(a, b) \geq 0$ if $a \neq b$. But in this case, since the analysis may not satisfy condition (3), symmetry, being zero distance apart may not be an equivalence relation, and so it may not be possible to divide the space into equivalence classes of exactly alike particulars.

Suppose, for example, that a chickpea has just two properties - roundness and brownness - whereas a macadamia nut has three properties - roundness, brownness, and deliciousness. Then the degree of dissimilarity between the chickpea and the macadamia nut according to this analysis is zero, since the chickpea has no properties at all which it does not have in common with the macadamia nut.

Nevertheless, we cannot treat the chickpea and the macadamia nut as if they are exactly the same, since as the macadamia nut has a property deliciousness - which the chickpea doesn't have, the degree of dissimmilarity of the macadamia nut to the chickpea is not zero, but one. In general, unless being dissimilar to degree zero is symmetric, we cannot treat particulars which are dissimilar to degree zero as if they are identical.

Note that if all particulars have the same fixed number of properties, then this analysis of degree of dissimilarity would satisfy symmetry, since the number of properties $a$ has not in common with $b$ would equal the number every particular has, minus the number $a$ has in common with $b$, and the 
number of properties $b$ has not in common with $a$ would equal the number every particular has, minus the number $b$ has in common with $a$, which is the same.

So the analysis of degree of dissimilarity between two particulars as the number of properties the first has not in common with the second does not entail that degree of dissimilarity is distance in a metric space. And if different particulars do not have the same number of properties not in common with each other, then it entails that degree of dissimilarity is not distance in a metric space, and that the two conceptions of degree of similarity are inconsonant with each other.

6.

If all particulars have the same fixed number of properties, then the degree of dissimilarity between two particulars could be defined as the fixed number of properties that all particulars have minus the number of properties in common between the two particulars. If the properties of a chickpea were brownness, roundness and yumminess and the properties of a pea were greenness, roundness and yuckiness, for example, then the degree of dissimilarity between the chickpea and the pea would be three minus one, or two.

This analysis of degree of dissimilarity would satisfy condition (1) because the degree of dissimilarity between a particular and itself would be the fixed number of properties every particular has minus the number of properties the particular has in common with itself or, in other words, the number of 
properties the particular has minus the number of properties the particular has, and so zero.

Likewise, this analysis would satisfy condition (2), according to which the distance between two different particulars is greater than zero, since two different particulars could not have as many as the fixed number of properties every particular has in common, since then they, in order to have properties with which to differ with the other on, would have to have more properties than the fixed number every particular has in common, which is impossible.

And condition (3), symmetry, would be satisfied by this analysis since the number of properties $a$ has in common with $b$ subtracted from the fixed number of properties every particular has must equal the number of properties $b$ has in common with $a$ subtracted from the fixed number every particular has, since the number of properties $a$ has in common with $b$ is the number of properties $b$ has in common with $a$.

Finally, since the number of properties two particulars have in common subtracted from the fixed number every particular has would be the number of properties the first has not in common with the second, and we know from the last section that the number of properties $a$ has not in common with $b$ plus the number $b$ has not in common with $c$ is at least as great as the number $a$ has not in common with $c$, it follows that condition (4), the triangle inequality, would be satisfied as well.

Moreover, since the number of properties two particulars have in common subtracted from the fixed number every particular has would be the number 
the first has not in common with the second, it follows that if all particulars had the same fixed number of properties, the analysis in this section would be equivalent to the analysis from section (5) as the number of properties the first particular has not in common with the second.

Likewise, since the highest number of properties any particular has would be the same as the fixed number of properties every particular has, it would follow that the analysis of degree of dissimilarity in this section would also be equivalent to the analysis of degree of dissimilarity between particulars from section (4) as the highest number of properties any particular has minus their number of properties in common.

So if all particulars have the same fixed number of properties, then it follows that the analysis of degree of dissimilarity between two particulars as the fixed number of properties every particular has minus the number of properties in common between the two particulars entails that degree of dissimilarity is distance in a metric space. Moreover, it follows that the three analyses discussed so far are equivalent, and all entail that degree of dissimilarity is distance in a metric space.

7.

Do all particulars have the same fixed number of properties? If every property has a corresponding negative property, then the answer is yes, since for each property a particular has, each other particular either has that same property, or else it has the corresponding negative property, and so for each 
property a particular has, each other particular has a corresponding property, and vice versa (McTaggart, 1921, 63).

But proponents of sparse conceptions of properties typically deny that every property has a corresponding negative property, which applies to a particular just in case the original property fails to apply to that particular. So proponents of sparse conceptions of properties typically cannot accept the argument from negative properties to the conclusion that all particulars have the same number of properties.

This is not an accidental feature of the view, since one of the main motivations for proponents of sparse conceptions of properties to deny that every property has a corresponding negative property is to allow sparse properties to feature in the analysis of similarity. If particulars resemble each other in virtue of having a property in common, it shouldn't follow that particulars which lack that property resemble each other in virtue of having its negation in common (Armstrong, 1978b, 23-24).

Moreover, another motivation for proponents of sparse conceptions of properties to deny that every property has a corresponding negative property is the consequence in question - that all particulars have the same number of properties. David Armstrong, for example, writes, 'If we restrict properties to positive properties, then it becomes a matter to be decided a posteriori if at all, whether two particulars have or have not the same number of properties. I suggest that this is a strong argument for restricting properties to positive properties.' Armstrong, 1978b, 24). 
So proponents of sparse conceptions of properties cannot argue that every particular has the same fixed number of properties on the basis that every property has a corresponding negative property. It does not follow that the thesis that all particular have the same fixed number of properties in common is inconsistent with a sparse conception of properties. But it does follow that there is no good reason for proponents of sparse conceptions of properties to accept the thesis.

So the thesis that all particulars have the same fixed number of properties is not entailed by the sparse conception of properties. If it is true, it is true merely a posteriori. And since the analyses above entail that degree of dissimilarity is distance in a metric space only in combination with the thesis that all particulars have the same fixed number of properties, whether according to those analyses degree of dissimilarity is distance in a metric space is at best a posteriori, and at worst false.

8.

If there is no fixed number of properties which every particular has, then we may still obtain a distance function by analysing the degree of dissimilarity between two particulars as the sum of the number of properties the first has not in common with the second and the number of properties the second has not in common with the first (in other words, as the cardinality of the symmetric difference of the set of properties of the first particular and the set of properties of the second particular). 
This analysis of degree of dissimilarity still satisfies condition (1), since no particular has any properties not in common with itself and vice versa, and so the analysis entails that the degree of dissimilarity between every particular and itself is zero plus zero, or still zero. And it still satisfies condition (2), since for any pair of different particulars, either the first has a property not in common with the second or the second has a property not in common with the first, and so the sum of their properties not in common is greater than zero.

But this analysis of degree of dissimilarity also satisfies condition (3), symmetry, since addition is commutative, and so for any two particulars, the number of properties the first has not in common with the second plus the number of properties the second has not in common with the first equals the number of properties the second has not in common with the first plus the number of properties the first has not in common with the second.

Finally, this analysis still satisfies condition (4), the triangle inequality. For for any property $a$ has not in common with $c$ or $c$ has not in common with $a$, either $a$ or $c$ also has that property not in common with $b$, or else either $a$ or $c$ does have that property in common with $b$, but then $b$ also has that property not in common with either $a$ or $c$, and so the sum of the dissimilarity between $a$ and $b$ and the dissimilarity between $b$ and $c$ is at least as great as the dissimilarity between $a$ and $c$.

But although this analysis satisfies the conditions of a distance function, it is still not a plausible analysis of degree of dissimilarity for two reasons. 
Firstly, it takes into account only the properties particulars have not in common, but not the properties they have in common. But intuitively, the degree of dissimilarity between two particulars should be less when they have more properties in common, and an adequate analysis of degree of dissimilarity should take this into account.

Secondly, insofar as there is a minimum degree of dissimilarity, which particulars with all properties in common have to each other, there should also be a maximum degree of dissimilarity, which particulars with no properties in common have to each other. Just as the degree of dissimilarity between peas in a pod, for example, has the minimum value of zero if they have all of their properties in common, the degree of dissimilarity between a raven and a writing desk, for example, should have a maximum value if they have none of their properties in common.

So although the analysis of the degree of dissimilarity between two particulars as the sum of the number of properties the first has not in common with the second and the number of properties the second has not in common with the first is a distance function, it is not a plausible analysis of degree of dissimilarity. The next section argues that the analysis of degree of dissimilarity as one minus proportion of properties in common, as well as defining a distance function, is a more plausible analysis. 
9.

A more plausible analysis of the degree of similarity between two particular is as their proportion of properties in common or, in other words, as their number of properties in common divided by the sum of the number of properties they have in common, the number of properties the first has not in common with the second, and the number of properties the second has not in common with the first (sometimes called the "Jaccard similarity" between the set of properties of the first and the set of properties of the second, due to its use by the botanist Paul Jaccard 1912, 39-40).

Since all particulars have some properties - there are no "bare" particulars - the number of properties in total between two particulars is never zero, and so their proportion of properties in common is always defined. Likewise, the degree of similarity between any particular and itself is always one, since because no particular has any properties not in common with itself, it is always the number of properties it has in common with itself divided by the number of properties it has in common with itself.

Since any two particulars have a nonnegative number of properties in common and a positive number of properties in total, the proportion of properties between any two particulars is greater than or equal to zero. And since the number of properties any two different particulars have in common is strictly less than the number of properties they have in total, the proportion of properties between any two different particulars is strictly less than one. 
So if the degree of similarity between two particulars is analysed as their proportion of properties in common, then it's natural to analyse the degree of dissimilarity between two particulars as one minus their degree of similarity or, in other words, one minus their number of properties in common divided by their number of properties in total (sometimes called the "Jaccard dissimilarity" between the set of properties of the first and the set of properties of the second (Levandowsky and Winter, 1971)).

This analysis of degree of dissimilarity meets condition (1), since the degree of similarity between a particular and itself is one, and so the degree of disimilarity between a particular and itself is one minus one or, in other words, zero. It meets condition (2), since the degree of similarity between two different particulars is strictly less than one, and so one minus the degree of similarity between any two different particulars is strictly greater than zero.

And it meets condition (3), symmetry, because any two particulars have the same number of properties in common as each other and the same number of properties in total as each other. Finally, it meets condition (4), the triangle inequality (for a basic but hard to summarize proof of this fact see Marczewski and Steinhaus, 1958, 321 and Levandowsky and Winter, 1971). So the analysis of degree of dissimilarity as one minus proportion of properties in common does entail that degree of dissimilarity is distance in a metric space. 
Finally, since it follows from the analysis of degree of similarity as proportion of properties in common that the degree of similarity between any two particulars is greater than or equal to zero, it follows from the analysis of degree of dissimilarity as one minus proportion of properties in common that the degree of dissimilarity between any two particulars is less than or equal to one. So the analysis of degree of dissimilarity as one minus proportion of properties in common entails that there is a maximum degree of dissimilarity.

So although several analyses of degree of similarity or dissimilarity do not entail that degree of dissimilarity is distance in a metric space, and entail that degree of dissimilarity is not distance in a metric space unless all particulars have the same fixed number of properties, at least one plausible analysis of dissimilarity - as proportion of properties in common - does entail that dissimilarity is distance in a metric space. Proponents of both conceptions could argue for this analysis.

10.

So far I have considered whether analysing degree of dissimilarity as a function of number of common and uncommon properties entails that the degree of dissimilarity between two particulars is their distance in a metric space. In this section I want to reverse this question and consider whether the thesis that the degree of dissimilarity between two particulars is their 
distance in a metric space entails an analysis of degree of dissimilarity as a function of number of properties in common and not in common.

The conception of degree of similarity as distance in a metric space does not entail the analysis of degree of dissimilarity as one minus proportion of properties in common, for at least two reasons. The first reason is that the analysis of degree of dissimilarity as one minus proportion of properties in common entails that there is a maximum degree of dissimilarity of one, which obtains between particulars which share none of their properties. But the conception of degree of similarity as distance in a metric space is compatible with there being no maximum degree of dissimilarity.

This point does not show that the conception of degree of dissimilarity as distance does not entail any analysis of dissimilarity in terms of number of common and uncommon properties. The analysis of the dissimilarity between two particulars as the number of properties the first has not in common with the second plus the number of properties the second has in common with the first, for example, is also compatible with their being no maximum degree of dissimilarity, since there is no maximum number of properties which a particular may have not in common with another.

Moreover, since to have no maximum distance a metric space must have an infinite number of elements, my raising this problem frustrates my desire to focus on the finite case and put problems to do with infinity aside. (Even if a metric space with a finite number of elements has a maximum distance 
of greater than one, it is plausible that it does not matter to rescale the distances by dividing them by that maximum, in which case the new maximum will be one.)

The second reason is that since the proportion of properties in common between any two particulars is a rational number, and one minus a rational number is a rational number, the analysis of degree of dissimilarity as one minus proportion of properties in common entails that the degree of dissimilarity between any two particulars is a rational number. But the conception of degree of similarity as distance in a metric space is compatible with the degree of dissimilarity between any two particulars being any real number.

It's compatible with the conception of degree of dissimilarity as distance that $a, b$, and $c$, for example, be arranged in a right triangle so that the degree of dissimilarity between $a$ and $b$ and between $b$ and $c$ is $\frac{1}{\sqrt{2}}$, and the degree of dissimilarity between $a$ and $c$ is one. But according to the analysis of degree of dissimilarity as one minus proportion of properties in common, the degree of dissimilarity between $a$ and $b$ could never be $\frac{1}{\sqrt{2}}$, as $\frac{1}{\sqrt{2}}$ is not a rational number.

This point does show that the conception of degree of dissimilarity as distance does not entail any analysis of dissimilarity as a function of number of properties in common and not in common. Since any function of number of properties in common and not in common has only integers in its domain, any function of number of properties in common and not in common has a 
countable range. But since a distance function is a function from a set of arbitrary pairs to the real numbers, it can have an uncountable range.

However, since for a distance function to have an uncountable range, it must also have an uncountable domain, my raising this point also frustrates my desire to focus on the finite case, and put problems to do with infinity aside. (Even if a metric space with a finite number of elements has irrational distances, it is plausible that it does not matter to rescale the distances so that all the values are rational, as long as doing so preserves the ordering of distances between pairs.)

But although these points essentially involve the infinite case, they also illustrate an important inconsonance between the conception of dissimilarity as distance in a metric space and the conception of similarity as a function of number of properties in common and not in common. The conception of dissimilarity as distance in a metric space is motivated by the vividness of the analogy between dissimilarity and spatial distance, and this analogy suggests degree of dissimilarity can vary continuously, just as space does.

This is not to say that degree of dissimilarity must vary continuously degree of dissimilarity with respect to electrical charge, for example, plausibly varies only discretely (see Eddon, 2006, 393-6 for a discussion of charge). It is just to say that degree of dissimilarity may vary continuously. But if degree of dissimilarity is proportion of properties not in common, degree of dissimilarity cannot vary continuously under any circumstances - and that is inconsonant with the analogy between distance and dissimilarity. 
Consider the analogy with probability. The analysis of probability as actual frequency has the advantage that it entails the axioms of the probability calculus, which embody part of our conception of probability. But the analysis of probabilities as actual frequencies is inconsonant with the conception embodied in the probability calculus, since the conception of probabilities as actual frequency entails that probabilities are rational, whereas the probability calculus suggests they may be irrational (Hajek, 1996, 224).

\section{1.}

The conception of degree of dissimilarity as distance in a metric space and the conception of similarity as a function of number of properties in common and not in common are logically independent. Moreover, the two conceptions of similarity are philosophically inconsonant - some plausible analyses in terms of properties do not define distance functions, and some plausible distance functions do not entail analyses in terms of properties.

In particular, the analysis of degree of dissimilarity as a strictly decreasing function of number of properties in common, and the analysis of the degree of dissimilarity between two particulars as the number of properties the first has not in common with the second, entail that degree of dissimilarity is distance in a metric space only if all particulars have the same fixed number of properties.

Moreover, if not all particulars have the same fixed number of properties, the analysis of degree of dissimilarity as a strictly decreasing function of 
number of properties in common, and the analysis of the degree of dissimilarity between two particulars as the number of properties the first has not in common with the second, both entail that degree of dissimilarity is not distance in a metric space.

The analysis of the degree of dissimilarity between two particulars as the sum of the number of properties the first has not in common with the second and the second has not in common with the first does entail that degree of dissimilarity is distance in a metric space. But it is not a plausible analysis of degree of dissimilarity, because it does not take into account number of properties in common.

The analysis of degree of dissimilarity as one minus proportion of properties in common is a plausible analysis, and does entail that degree of dissimilarity is distance in a metric space. But it is considerably less general than the conception of degree of dissimilarity as distance in a metric space, and also inconsonant with the analogy between dissimilarity and spatial distance which supports that conception.

So although proponents of both conceptions of degree of dissimilarity could argue for the analysis of degree of dissimilarity as proportion of properties not in common, it is not clear that they should. It may be better for proponents of the conception as distance in a metric space to justify their position in 
another way, or for proponents of the conception as a function of properties in common and not in common to adopt a different analysis ${ }^{1}$

\section{REFERENCES}

Armstrong, D. (1978a). Nominalism and Realism. Cambridge: Cambridge University Press.

Armstrong, D. (1978b). A Theory of Universals. Cambridge: Cambridge University Press.

Armstrong, D. (1989). Universals. Boulder: Westview Press.

Armstrong, D. (1997). Against Ostrich Nominalism, in Huw Mellor and Alex Oliver (eds), Properties. Oxford: Oxford University Press.

Bigelow, J. (1976). Possible Worlds Foundations for Probability, Journal of Philosophical Logic 5/3: 299-320.

Bigelow, J. (1977). Semantics of Probability, Synthese 36/4: 459-472.

Blumson, B. (2014). Resemblance and Representation. Cambridge: Open Book Publishers.

Dorr, C. and Hawthorne, J. (2013). Naturalness, in K. Bennett and D. Zimmerman (eds), Oxford Studies in Metaphysics, Vol. 8. Oxford: Oxford University Press, pp. 2-77.

\footnotetext{
${ }^{1}$ I'm grateful to Jay Garfield, Raamy Majeed, Nicholas Silins, Hsueh Qu and Weng Hong Tang for reading drafts of this paper. I'm also grateful to audiences at the Australian Metaphysics Conference, the Australasian Association of Logic, and Lingnan University.
} 
Eddon, M. (2006). Armstrong on Quantities and Resemblance, Philosophical Studies 136/3: 385-404.

Goodman, N. (1972). Seven Strictures on Similarity, Problems and Projects. Indianapolis: Bobbs-Merril.

Hajek, A. (1996). "Mises Redux" - Redux: Fifteen Arguments Against Finite Frequentism, Erkenntnis 45/2-3: 209-227.

Jaccard, P. (1912). The Distribution of the Flora in the Alpine Zone, New Phytologist 11/2: 37-50.

Kroedel, T. and Huber, F. (2013). Counterfactual Dependence and Arrow, Nous 47/3: 453-466.

Levandowsky, M. and Winter, D. (1971). Distance Between Sets, Nature 234/5323: 34-35.

Lewis, D. (1973). Counterfactuals. Oxford: Blackwell.

Lewis, D. (1983). New Work for a Theory of Universals, Australasian Journal of Philosophy 61/4: 343-377.

Lewis, D. (1986). On the Plurality of Worlds. Oxford: Blackwell.

Marczewski, E. and Steinhaus, H. (1958). On a Certain Distance of Sets and the Corresponding Distance of Functions, Colloquium Mathematicae 6/1: 319-327.

McTaggart, J. (1921). The Nature of Existence, Vol. 1. Cambridge: Cambridge University Press.

Niiniluoto, I. (1987). Truthlikeness. Dordrecht: Kluwer.

Oddie, G. (1986). Likeness to Truth. Dordrecht: Kluwer. 
Oliver, A. (1996). The Metaphysics of Properties, Mind 105/417: 1-80.

Paseau, A. (2012). Resemblance Theories of Properties, Philosophical Studies 157/3: 361-382.

Rodriguez-Pereyra, G. (2002). Resemblance Nominalism. Oxford: Oxford University Press.

Schaffer, J. (2004). Two Conceptions of Sparse Properties, Pacific Philosophical Quarterly 85/1: 92-102.

Sobel, H. (1970). Utilitarianisms: Simple and General, Inquiry 13/1-4: 394449.

Suppes, P., Krantz, D., Luce, D. and Tversky, A. (1989). Foundations of Measurement, Vol. 2. San Diego: Academic Press.

Tversky, A. (1977). Features of Similarity, Psychological Review 84/4: 327352.

Williamson, T. (1988). First-Order Logics for Comparative Similarity., Notre Dame Journal of Formal Logic 29/4: 457-481.

National University of Singapore, Singapore 